\title{
SYMMETRIC OPERATORS WITH TWICE CONTINUOUSLY DIFFERENTIABLE SPECTRAL FUNCTIONS
}

\author{
RICHARD C. GILBERT ${ }^{1}$
}

In a previous article [2] it was shown that if $A$ is a simple closed symmetric operator with deficiency indices $(1,1)$ in a Hilbert space $H$ and if $A$ has a selfadjoint extension $A_{0}$ in $H$ with a discrete spectrum, then every selfadjoint extension and every minimal selfadjoint dilation $A^{+}$of $A$ has spectral multiplicity 1. Further, it was possible to make certain statements about the spectrum of $A^{+}$. In the present article it is shown that if $A$ has a selfadjoint extension $A_{0}$ with a twice continuously differentiable spectral function, whose derivative is always positive, then there are minimal selfadjoint dilations $A^{+}$of $A$ for which the spectral multiplicity is two; indeed, there are minimal selfadjoint dilations $A^{+}$which are unitarily equivalent to the multiplication operator in $L^{2}(-\infty, \infty) \oplus L^{2}(-\infty, \infty)$. As in [2] the proof of these facts depends upon obtaining an expansion theorem which is valid for each of the selfadjoint extensions or dilations $A^{+}$. This expansion theorem is the analog of the well-known expansion theorems for linear ordinary differential operators; however, it is necessary in deriving it to overcome the fact that, unlike a linear ordinary differential operator, there does not necessarily exist a basis of entire functions for the solutions of the equation $A f=\lambda f$. After the expansion theorem is obtained, it is used to define a unitary map of $H^{+}$(the space in which $A^{+}$acts) onto $L^{2}(-\infty, \infty) \oplus L^{2}(-\infty, \infty)$. The same map takes $A^{+}$into the multiplication operator in $L^{2}(-\infty, \infty)$ $\oplus L^{2}(-\infty, \infty)$.

Throughout this article we shall adhere to the notation of [2], except that we shall use the symbol $Q(\lambda)$ in place of $Q_{1}(\lambda)$.

We begin with a lemma on the limit of a function analytic in the upper half-plane as one approaches the real axis.

Lemma. Suppose for all nonreal $\lambda, \theta(\lambda)=\int_{-\infty}^{\infty}(t-\lambda)^{-1} d \rho(t)$, where $\rho(t)$ is a nondecreasing bounded function which is twice continuously differentiable for all $t$. Then, $\theta(\lambda)$ may be defined continuously down to the

Presented to the Society, April 26,1969; received by the editors February 10, 1969.

${ }^{1}$ This work was performed in part while the author was on a research leave granted by California State College at Fullerton. 
real axis. For a point $\xi$ on the real axis we shall have

$$
\begin{aligned}
& \operatorname{Re} \theta(\xi)=P \int_{-\infty}^{\infty}(t-\xi)^{-1} d \rho(t), \\
& \operatorname{Im} \theta(\xi)=\pi \rho^{\prime}(\xi),
\end{aligned}
$$

where $P$ stands for Cauchy principal value.

Proof. Let $f(\xi, t)=\left[\rho^{\prime}(t)-\rho^{\prime}(\xi)\right](t-\xi)^{-1}$ if $t \neq \xi$, and let $f(\xi, t)$ $=p^{\prime \prime}(t)$ if $t=\xi$. Then, $f(\xi, t)$ is a continuous function, and $\rho^{\prime}(t)=\rho^{\prime}(\xi)$ $+(t-\xi) f(\xi, t)$.

Now,

$$
\begin{aligned}
& P \int_{-\infty}^{\infty}(t-\xi)^{-1} d \rho(t) \\
& \quad=\int_{-\infty}^{\xi-1}(t-\xi)^{-1} d \rho(t)+\int_{\xi+1}^{\infty}(t-\xi)^{-1} d \rho(t)+\int_{\xi-1}^{\xi+1} f(\xi, t) d t \\
& \quad=\int_{-\infty}^{-1} u^{-1} \rho^{\prime}(u+\xi) d u+\int_{1}^{\infty} u^{-1} \rho^{\prime}(u+\xi) d u+\int_{-1}^{1} f(\xi, u+\xi) d u
\end{aligned}
$$

From this last expression it is not difficult to see that $P \int_{-\infty}^{\infty}(t-\xi)^{-1} d \rho(t)$ exists and is a continuous function of $\xi$. If $\lambda=\xi+i \eta$, then

$$
\begin{aligned}
\operatorname{Re} \theta(\lambda)= & \int_{-\infty}^{\infty}(t-\xi)\left[(t-\xi)^{2}+\eta^{2}\right]^{-1} d \rho(t) \\
= & \int_{-\infty}^{-1}\left[u+\left(\xi_{0}-\xi\right)\right]\left\{\left[u+\left(\xi_{0}-\xi\right)\right]^{2}+\eta^{2}\right\}^{-1} \rho^{\prime}\left(u+\xi_{0}\right) d u \\
& +\int_{1}^{\infty}\left[u+\left(\xi_{0}-\xi\right)\right]\left\{\left[u+\left(\xi_{0}-\xi\right)\right]^{2}+\eta^{2}\right\}^{-1} \rho^{\prime}\left(u+\xi_{0}\right) d u \\
& +(1 / 2) \rho^{\prime}(\xi) \log \left\{\left[1-\left(\xi-\xi_{0}\right)\right]^{2}+\eta^{2}\right\}\left\{\left[1+\left(\xi-\xi_{0}\right)\right]^{2}+\eta^{2}\right\}^{-1} \\
& +\int_{-1}^{1} f\left(\xi, u+\xi_{0}\right) d u-\eta^{2} \int_{-1}^{1}\left\{\left[u-\left(\xi-\xi_{0}\right)\right]^{2}+\eta^{2}\right\}^{-1} \\
& \cdot f\left(\xi, u+\xi_{0}\right) d u .
\end{aligned}
$$

From this last expression it is not difficult to see that $\lim _{\lambda \rightarrow \xi_{0}} \operatorname{Re} \theta(\lambda)$ $=P \int_{-\infty}^{\infty}\left(t-\xi_{0}\right)^{-1} d \rho(t)$. Hence, $\operatorname{Re} \theta(\lambda)$ can be defined continuously down to the real axis, and itsvalue on the real axis is $P \int_{-\infty}^{\infty}(t-\xi)^{-1} d \rho(t)$.

Now, 


$$
\begin{aligned}
\operatorname{Im} \theta(\lambda)= & \eta \int_{-\infty}^{\infty}\left[(t-\xi)^{2}+\eta^{2}\right]^{-1} d \rho(t) \\
= & \eta \int_{-\infty}^{-1}\left\{\left[u-\left(\xi-\xi_{0}\right)\right]^{2}+\eta^{2}\right\}^{-1} \rho^{\prime}\left(u+\xi_{0}\right) d u \\
& +\eta \int_{1}^{\infty}\left\{\left[u-\left(\xi-\xi_{0}\right)\right]^{2}+\eta^{2}\right\}^{-1} \rho^{\prime}\left(u+\xi_{0}\right) d u \\
& +\rho^{\prime}(\xi)\left\{\tan ^{-1} \eta^{-1}\left[1-\left(\xi-\xi_{0}\right)\right]+\tan ^{-1} \eta^{-1}\left[1+\left(\xi-\xi_{0}\right)\right]\right\} \\
& +\eta \int_{-1}^{1}\left[u-\left(\xi-\xi_{0}\right)\right]\left\{\left[u-\left(\xi-\xi_{0}\right)\right]^{2}+\eta^{2}\right\}-1 f\left(\xi, u+\xi_{0}\right) d u .
\end{aligned}
$$

From this expression it is not difficult to see that $\lim _{\lambda \rightarrow \xi_{0}} \operatorname{Im} \theta(\lambda)$ $=\pi \rho^{\prime}\left(\xi_{0}\right)$. Since $\rho^{\prime}(\xi)$ is continuous, we see that $\operatorname{Im} \theta(\lambda)$ can be defined continuously down to the real axis, and its value on the real axis is $\pi \rho^{\prime}(\xi)$.

This completes the proof of the lemma.

Let $A$ be a simple closed symmetric operator with deficiency indices $(1,1)$ in the Hilbert space $H$. Let $\lambda_{0}$ be a complex number with positive imaginary part, and let $g_{0}$ be an element of norm 1 in the deficiency subspace of $A$, corresponding to $\lambda_{0}^{-}$. (Here $\lambda_{0}^{-}$stands for complex conjugate.) Suppose that $A_{0}$ is a selfadjoint extension of $A$ in $H$. Since $A$ is simple, $g_{0}$ is a generating element for $A_{0}$. (See Achieser and Glasmann [1, §81, Theorem 2].) Hence, $A_{0}$ is unitarily equivalent to the multiplication operator in $L_{\rho_{0}}^{2}$, where $\rho_{0}(t)$ $=\left(E_{0}(t) g_{0}, g_{0}\right)$ and $E_{0}(t)$ is the spectral function of $A_{0}$. By $S$ we shall denote those elements $f$ in $H$ whose transforms $F(t)$ in $L_{\rho_{0}}^{2}$ are continuously differentiable functions. $S$ is dense in $H$.

Suppose that $R_{0}(\lambda)$ is the resolvent of $A_{0}$. Let $g(\lambda)=g_{0}+\left(\lambda-\lambda_{0}\right)$ - $R_{0}(\lambda) g_{0}$, and let $Q(\lambda)=i \operatorname{Im} \lambda_{0}+\left(\lambda-\lambda_{0}\right)\left(g_{0}, g\left(\lambda^{-}\right)\right)$. As is indicated in [2, Lemma 2], for $\operatorname{Im} \lambda \neq 0$ the generalized resolvent $R(\lambda)$ of $A$ corresponding to a selfadjoint extension or dilation $A^{+}$of $A$ has the form

$$
R(\lambda)=R_{0}(\lambda)-[\theta(\lambda)+Q(\lambda)]^{-1}\left(\cdot, g\left(\lambda^{-}\right)\right) g(\lambda),
$$

where $\theta(\lambda)$ is an analytic function for $\operatorname{Im} \lambda \neq 0$ which has nonnegative imaginary part in the upper half-plane. $R(\lambda)$ corresponds to a selfadjoint extension in the space $H$ if and only if $\theta(\lambda)$ is identically equal to a constant $\theta,-\infty<\theta \leqq+\infty$.

TheOREM 1 (EXPANsion THEOREM). Suppose $\rho_{0}(t)$ is twice continuously differentiable and that $\rho_{0}^{\prime}(t)>0$ for all $t$. Let $A^{+}$be a selfadjoint 
extension of $A$ in $H$ or a selfadjoint dilution of $A$ in $H^{+} \supset H$. Let $E^{+}(\lambda)$ be the spectral function of $A^{+}$. Suppose the function $\theta(\lambda)$ of equation (1) has the form $\theta(\lambda)=a+b \lambda+\int_{-\infty}^{\infty}(t-\lambda)^{-1} d \rho(t)$, where $b \geqq 0$, a is real, $\rho(t)$ is nondecreasing, bounded and twice continuously differentiable. Then for any numbers $\alpha, \beta, \alpha<\beta$, and for any two elements $f, h \in S$,

$$
\left(\left[E^{+}(\beta)-E^{+}(\alpha)\right] f, h\right)=\int_{\alpha}^{\beta} \sum_{i, j=1}^{2} C_{i}(f ; \xi)\left[C_{j}(h ; \xi)\right]-\delta_{i j}(\xi) d \xi,
$$

where

$$
\begin{aligned}
C_{1}(f ; \xi)= & \left(f, g_{0}\right)+\left(\xi-\lambda_{0}^{-}\right) P \int_{-\infty}^{\infty}(t-\xi)^{-1} d\left(f, E_{0}(t) g_{0}\right), \\
C_{2}(f ; \xi)= & \pi\left(\xi-\lambda_{0}^{-}\right)\left(f, E_{0}(\xi) g_{0}\right)^{\prime}, \quad \text { where the prime stands for } \\
\text { differentiation, } & \\
\delta_{11}(\xi)= & B(\xi) / \pi\left[A^{2}(\xi)+B^{2}(\xi)\right], \\
\delta_{12}(\xi)= & \delta_{21}(\xi)=-A(\xi) / \pi\left[A^{2}(\xi)+B^{2}(\xi)\right], \\
\delta_{22}(\xi)= & 1 / \pi^{2}\left|\xi-\lambda_{0}\right|^{2} \rho_{0}^{\prime}(\xi)-B(\xi) / \pi\left[A^{2}(\xi)+B^{2}(\xi)\right], \\
A(\xi)= & P \int_{-\infty}^{\infty}(t-\xi)^{-1} d \rho(t)+a+b \xi+\left(\xi-\operatorname{Re} \lambda_{0}\right) \\
& +\left|\xi-\lambda_{0}\right|^{2} P \int_{-\infty}^{\infty}(t-\xi)^{-1} d \rho_{0}(t), \\
B(\xi)= & \pi\left[\rho^{\prime}(\xi)+\left|\xi-\lambda_{0}\right|^{2} \rho_{0}{ }^{\prime}(\xi)\right] .
\end{aligned}
$$

Proof. By the process of polarization applied to $\left(E_{0}(t) f, h\right)$, we see that

$$
\begin{aligned}
\left(R_{0}(\lambda) f, h\right)= & \int_{-\infty}^{\infty}(t-\lambda)^{-1} d\left(E_{0}(t) f, h\right) \\
= & (1 / 4) \int_{-\infty}^{\infty}(t-\lambda)^{-1} d\left(E_{0}(t)(f+h), f+h\right) \\
& -(1 / 4) \int_{-\infty}^{\infty}(t-\lambda)^{-1} d\left(E_{0}(t)(f-h), f-h\right) \\
& +(i / 4) \int_{-\infty}^{\infty}(t-\lambda)^{-1} d\left(E_{0}(t)(f+i h), f+i h\right) \\
& -(i / 4) \int_{-\infty}^{\infty}(t-\lambda)^{-1} d\left(E_{0}(t)(f-i h), f-i h\right) .
\end{aligned}
$$


Since $\left(E_{0}(t)(f+h), f+h\right)=\int_{-\infty}^{t}|F(\xi)+H(\xi)|{ }^{2} d \rho_{0}(\xi)$, and since $F(\xi)$, $H(\xi)$ are continuously differentiable, we see that $\left(E_{0}(t)(f+h), f+h\right)$ $\in C^{2}$. The same is true for the other terms in (3). It follows from the lemma, then, that $\left(R_{0}(\lambda) f, h\right)$ can be defined continuously down to the real axis and that at a point $\xi$ on the real axis,

$$
\left(R_{0}(\xi) f, h\right)=P \int_{-\infty}^{\infty}(t-\xi)^{-1} d\left(E_{0}(t) f, h\right)+i \pi\left(E_{0}(\xi) f, h\right)^{\prime} .
$$

Since $\left(E_{0}(t) f, \quad h\right)=\int_{-\infty}^{t} F(u)[H(u)]-\rho_{0}^{\prime}(u) d u$, equation (4) can be written

$$
\begin{aligned}
\left(R_{0}(\xi) f, h\right)= & P \int_{-\infty}^{\infty}(t-\xi)^{-1} F(t)[H(t)]^{-} \rho_{0}^{\prime}(t) d t \\
& +i \pi F(\xi)[H(\xi)]^{-} \rho_{0}^{\prime}(\xi) .
\end{aligned}
$$

One can show similarly that $\left(f, g\left(\lambda^{-}\right)\right)$and $(g(\lambda), h)$ can be defined continuously down to the real axis and that at a point $\xi$ on the real axis,

$$
\begin{aligned}
(f, g(\xi)) & =C_{1}(f ; \xi)+i C_{2}(f ; \xi), \\
(g(\xi), h) & =\left[C_{1}(h ; \xi)\right]^{-}+i\left[C_{2}(h ; \xi)\right]^{-} .
\end{aligned}
$$

Since $Q(\lambda)=i \operatorname{Im} \lambda_{0}+\left(\lambda-\lambda_{0}\right)+\left|\lambda-\lambda_{0}\right|^{2} \int_{-\infty}^{\infty}(t-\lambda)^{-1} d \rho_{0}(t)$, and since $\theta(\lambda)=a+b \lambda+\int_{-\infty}^{\infty}(t-\lambda)^{-1} d \rho(t)$, it follows from the lemma that $\theta(\lambda)+Q(\lambda)$ can be defined continuously down to the real axis and that at a point $\xi$ on the real axis, $\theta(\xi)+Q(\xi)=A(\xi)+i B(\xi)$. Since $\rho_{0}^{\prime}(\xi)>0$ for all $\xi,[\theta(\lambda)+Q(\lambda)]^{-1}$ will be continuous down to the real axis, and on the real axis,

$$
[\theta(\xi)+Q(\xi)]^{-1}=[A(\xi)+i B(\xi)]^{-1} .
$$

From equation (1) and equations (5), (6), (7), (8), we now see that $(R(\lambda) f, h)$ can be defined continuously down to the real axis, where $R(\lambda)$ is the generalized resolvent corresponding to $A^{+}$, and we see that on the real axis,

$$
\begin{aligned}
(R(\xi) f, h)= & P \int_{-\infty}^{\infty}(t-\xi)^{-1} F(t)[H(t)]^{-} \rho_{0}^{\prime}(t) d t \\
& +i \pi F(\xi)[H(\xi)]^{-} \rho_{0}^{\prime}(\xi) \\
& -[A(\xi)+i B(\xi)]^{-1}\left[C_{1}(f ; \xi)+i C_{2}(f ; \xi)\right] \\
& \cdot\left\{\left[C_{1}(h ; \xi)\right]^{-}+i\left[C_{2}(h ; \xi)\right]-\right\} .
\end{aligned}
$$

The Stieltjes inversion formula states that 


$$
\begin{array}{r}
\left(\left[(1 / 2)\left\{E^{+}(\beta)+E^{+}(\beta+0)\right\}-(1 / 2)\left\{E^{+}(\alpha)+E^{+}(\alpha+0)\right\}\right] f, h\right) \\
=(2 \pi i)^{-1} \lim _{\eta \rightarrow+0} \int_{\alpha}^{\beta}\left[(R(\xi+i \eta) f, h)-(R(\xi+i \eta) h, f)^{-}\right] d \xi .
\end{array}
$$

Since the terms under the integral sign are continuous down to the real axis, we may take the limit under the integral sign. Using equation (9), we obtain that

$$
\begin{aligned}
(2 \pi i)^{-1} \lim _{\eta \rightarrow+0}[( & \left.R(\xi+i \eta) f, h)-(R(\xi+i \eta) h, f)^{-}\right] \\
= & F(\xi)[H(\xi)]-\rho_{0}^{\prime}(\xi)+\sum_{i, j=1}^{2} C_{i}(f ; \xi)\left[C_{j}(h ; \xi)\right]-d_{i j}(\xi),
\end{aligned}
$$

where

$$
\begin{aligned}
& d_{i i}(\xi)=-d_{22}(\xi)=B(\xi) / \pi\left[A^{2}(\xi)+B^{2}(\xi)\right] \\
& d_{12}(\xi)=d_{21}(\xi)=-A(\xi) / \pi\left[A^{2}(\xi)+B^{2}(\xi)\right] .
\end{aligned}
$$

Now,

$$
\left(f, E_{0}(\xi) g_{0}\right)=\int_{-\infty}^{\xi} F(t) d \rho_{0}(t)
$$

Hence,

$$
F(\xi)=\left(f, E_{0}(\xi) g_{0}\right)^{\prime} / \rho_{0}^{\prime}(\xi)=C_{2}(f ; \xi) / \pi\left(\xi-\lambda_{0}^{-}\right) \rho_{0}^{\prime}(\xi) .
$$

Similarly,

$$
H(\xi)=C_{\mathbf{2}}(h ; \xi) / \pi\left(\xi-\lambda_{0}^{-}\right) \rho_{0}^{\prime}(\xi) .
$$

Substituting these expressions for $F(\xi)$ and $H(\xi)$ into (11) and collecting terms, we obtain that

$$
\begin{array}{r}
(2 \pi i)^{-1} \lim _{\eta \rightarrow+0}\left[(R(\xi+i \eta) f, h)-(R(\xi+i \eta) h, f)^{-}\right] \\
=\sum_{i, j=1}^{2} C_{i}(f ; \xi)\left[C_{j}(h ; \xi)\right]-\delta_{i j}(\xi) .
\end{array}
$$

The theorem then follows from equations (10) and (12).

THEOREM 2. Suppose the conditions of Theorem 1 are valid, with the additional assumption that if $A^{+}$is a selfadjoint dilation of $A$, then it is minimal (i.e., it is reduced by no nontrivial subspace of $H^{+} \ominus H$ ). If $\rho^{\prime}(\xi)>0$ for all $\xi$, then $A^{+}$is unitarily equivalent to the multiplication operator in $L^{2}(-\infty, \infty) \oplus L^{2}(-\infty, \infty)$; hence, the spectral multiplicity of $A^{+}$is two, and the whole real axis is in the continuous spectrum. On 
the other hand, if $\rho^{\prime}(\xi) \equiv 0$, then $A^{+}$is unitarily equivalent to the multiplication operator in $L^{2}(-\infty, \infty)$; hence, the spectral multiplicity of $\mathrm{A}^{+}$ is one, and again the whole real axis is in the continuous spectrum. (In particular, the latter statement holds if $A^{+}$is a selfadjoint extension of $A$ in $H$.)

Proof. Let $Z$ be the set of all elements in $H^{+}$of the form $\left[E^{+}(\beta)-E^{+}(\alpha)\right] f$, where $\alpha, \beta$ are any two numbers such that $\alpha<\beta$, and $f$ is any element in $H$. Let $Z_{1}$ be the set of elements of the same form except that $f$ is any element of $S$. Since $A^{+}$is minimal, it is known that $H^{+}$is the closed linear hull of $Z$. (See Naimark [3].) Since $S$ is dense in $H, H^{+}$is also the closed linear hull of $Z_{1}$.

By completing the square on the integrand, we can write equation (2) in the form

$$
\begin{aligned}
\left(\left[E^{+}(\beta)-\right.\right. & \left.\left.E^{+}(\alpha)\right] f, h\right) \\
= & \int_{\alpha}^{\beta}\left[C_{1}(f ; \xi) \delta_{11}^{1 / 2}(\xi)+\delta_{12}(\xi) \delta_{11}^{-1 / 2}(\xi) C_{2}(f ; \xi)\right] \\
& \cdot\left[C_{1}(h ; \xi) \delta_{11}^{1 / 2}(\xi)+\delta_{12}(\xi) \delta_{11}^{-1 / 2}(\xi) C_{2}(h ; \xi)\right]^{-} d \xi \\
& +\int_{\alpha}^{\beta} C_{2}(f ; \xi)\left[C_{2}(h ; \xi)\right]^{-}\left[\delta_{11}(\xi) \delta_{22}(\xi)-\delta_{12}^{2}(\xi)\right] \delta_{11}^{-1}(\xi) d \xi .
\end{aligned}
$$

Let us note that

$$
\delta_{11}(\xi) \delta_{22}(\xi)-\delta_{12}^{2}(\xi)=\rho^{\prime}(\xi)\left\{\pi^{2}\left[A^{2}(\xi)+B^{2}(\xi)\right] \cdot\left|\xi-\lambda_{0}\right|^{2} \rho_{0}^{\prime}(\xi)\right\}^{-1}
$$

If $\rho^{\prime}(\xi)>0$ for all $\xi$, then $\delta_{11}(\xi) \delta_{22}(\xi)-\delta_{12}^{2}(\xi)>0$ for all $\xi$. In this case we define an operator $V$ on $Z_{1}$ into $L^{2}(-\infty, \infty) \oplus L^{2}(-\infty, \infty)$ as follows:

$$
\begin{array}{r}
V\left[E^{+}(\beta)-E^{+}(\alpha)\right] f=\chi_{[\alpha, \beta)}(\xi)\left[C_{1}(f ; \xi) \delta_{11}^{1 / 2}(\xi)+\delta_{12}(\xi) \delta_{11}^{-1 / 2}(\xi) C_{2}(f ; \xi),\right. \\
\left.C_{2}(f ; \xi)\left\{\delta_{11}(\xi) \delta_{22}(\xi)-\delta_{12}^{2}(\xi)\right\}^{1 / 2} \delta_{11}^{-1 / 2}(\xi)\right],
\end{array}
$$

where $\chi_{[\alpha, \beta)}(\xi)$ is the characteristic function of $[\alpha, \beta)$. From (13) we see that

$$
\left\|V\left[E^{+}(\beta)-E^{+}(\alpha)\right] f\right\|=\left\|\left[E^{+}(\beta)-E^{+}(\alpha)\right] f\right\| .
$$

We now extend $V$ linearly to the linear hull of $Z_{1}$, and by continuity to all of $\mathrm{H}^{+} . V$ is then an isometry of $H^{+}$into $L^{2}(-\infty, \infty)$ $\oplus L^{2}(-\infty, \infty)$.

$V$ is in fact onto, because the linear hull of the set $V Z_{1}$ is dense in 
$L^{2}(-\infty, \infty) \oplus L^{2}(-\infty, \infty)$. To see this, suppose $\left[k_{1}(\xi), k_{2}(\xi)\right]$ $\in L^{2}(-\infty, \infty) \oplus L^{2}(-\infty, \infty)$ and that $\left[k_{1}(\xi), k_{2}(\xi)\right]$ is perpendicular to $V Z_{1}$. Then,

$$
\begin{aligned}
& \int_{[\alpha, \beta)}\left\{\left[C_{1}(f ; \xi) \delta_{11}^{1 / 2}(\xi)+\delta_{12}(\xi) \delta_{11}^{-1 / 2}(\xi) C_{2}(f ; \xi)\right] k_{1}^{-}(\xi)\right. \\
& \left.\quad+C_{2}(f ; \xi)\left[\delta_{11}(\xi) \delta_{22}(\xi)-\delta_{12}^{2}(\xi)\right]^{1 / 2} \delta_{11}^{-1 / 2}(\xi) k_{2}^{-}(\xi)\right\} d \xi=0
\end{aligned}
$$

for all $\alpha, \beta, \alpha<\beta$, and for all $f \in S$. Let $(\gamma, \delta)$ be an arbitrary interval, and let $\left\{\gamma_{n}\right\},\left\{\delta_{n}\right\}$ be sequences of numbers such that $\gamma>\gamma_{1}>\gamma_{2}$ $>\cdots \rightarrow-\infty$, and $\delta<\delta_{1}<\delta_{2}<\cdots \rightarrow+\infty$. Let $F_{n}(t)$ sbe any function in $L_{\rho_{0}}^{2}$ which is continuously differentiable, vanishes in $\left[\gamma_{n}, \delta_{n}\right]$ and is positive outside $\left[\gamma_{n}, \delta_{n}\right]$. Let $f_{n}$ be the element in $S$ of which $F_{n}(t)$ is the transform, and let $g_{n}=f_{n} /\left(f_{n}, g_{0}\right)$. Then, if $G_{n}(t)$ is the transform of $g_{n}$, we have that $\int_{-\infty}^{\infty} G_{n}(t) d \rho_{0}(t)=1$. Since $\left(g_{n}, E_{0}(\xi) g_{0}\right)$ $=\int_{-\infty}^{\xi} G_{n}(t) d \rho_{0}(t), C_{2}\left(g_{n} ; \xi\right)=\pi\left(\xi-\lambda_{0}^{-}\right)\left(g_{n}, E_{0}(\xi) g_{0}\right)^{\prime}=0$ for $\gamma<\xi<\delta$. By equation (6), $C_{1}\left(g_{n} ; \xi\right)=\left(g_{n}, g(\xi)\right)$ for $\gamma<\xi<\delta$. Now, $\lim _{n \rightarrow \infty}\left(g_{n}, g(\xi)\right)=1$ uniformly for $\gamma<\xi<\delta$, because for $\gamma<\xi<\delta$,

$$
\begin{aligned}
& \left|\left(g_{n}, g(\xi)\right)-1\right|=\mid \int_{-\infty}^{\gamma_{n}} G_{n}(t)\left(t-\lambda_{0}^{-}\right)(t-\xi)^{-1} d \rho_{0}(t) \\
& \quad+\int_{\delta_{n}}^{\infty} G_{n}(t)\left(t-\lambda_{0}^{-}\right)(t-\xi)^{-1} d \rho_{0}(t) \\
& \quad-\int_{-\infty}^{\gamma_{n}} G_{n}(t) d \rho_{0}(t)-\int_{\delta_{n}}^{\infty} G_{n}(t) d \rho_{0}(t) \mid \\
& \quad \leqq \int_{-\infty}^{\gamma_{n}} G_{n}(t)\left|\xi-\lambda_{0}^{-}\right||t-\xi|^{-1} d \rho_{0}(t) \\
& \quad+\int_{\delta_{n}}^{\infty} G_{n}(t)\left|\xi-\lambda_{0}^{-}\right||t-\xi|^{-1} d \rho_{0}(t) \\
& \quad \leqq K / \min \left\{\delta_{n}-\delta, \gamma-\gamma_{n}\right\},
\end{aligned}
$$

where $K$ is a constant. Thus, we see that for the sequence $\left\{g_{n}\right\}$, $C_{2}\left(g_{n} ; \xi\right)=0$ for $\gamma<\xi<\delta$, and $\lim _{n \rightarrow \infty} C_{1}\left(g_{n} ; \xi\right)=1$ uniformly for $\gamma<\xi<\delta$. If we substitute the elements $g_{n}$ into equation (15) and take the limit as $n \rightarrow \infty$, we shall obtain that $\int_{[\alpha, \beta)} \delta_{11}^{1 / 2}(\xi) k_{1}^{-}(\xi) d \xi=0$. Since this equation is true for all $\alpha, \beta$, it follows that $\delta_{11}^{1 / 2}(\xi) k_{1}(\xi)=0$ a.e., and therefore $k_{1}(\xi)=0$ a.e. Equation (15) thus becomes

$$
\int_{\{\alpha, \beta)} C_{2}(f ; \xi)\left\{\delta_{11}(\xi) \delta_{22}(\xi)-\delta_{12}^{2}(\xi)\right\}^{1 / 2} \delta_{11}^{-1 / 2}(\xi) \overrightarrow{k_{2}}(\xi) d \xi=0
$$


for all $\alpha, \beta, \alpha<\beta$, and for all $f \in S$. Now,

$$
C_{2}\left(g_{0} ; \xi\right)=\pi\left(\xi-\lambda_{0}^{\overline{0}}\right)\left(g_{0}, E_{0}(\xi) g_{0}\right)^{\prime}=\pi\left(\xi-\lambda_{0}^{\overline{0}}\right) \rho_{0}^{\prime}(\xi) .
$$

Taking $f=g_{0}$ in equation (16), we see, then, that

$$
\pi\left(\xi-\lambda_{0}^{-}\right) \rho_{0}^{\prime}(\xi)\left\{\delta_{11}(\xi) \delta_{22}(\xi)-\delta_{12}^{2}(\xi)\right\}^{1 / 2} \delta_{11}^{-1 / 2}(\xi) \overrightarrow{k_{2}}(\xi)=0 \text { a.e., }
$$

and hence $k_{2}(\xi)=0$ a.e. Thus, we have shown that if $\left[k_{1}(\xi), k_{2}(\xi)\right]$ $\in L^{2}(-\infty, \infty) \oplus L^{2}(-\infty, \infty)$ and if $\left[k_{1}(\xi), k_{2}(\xi)\right]$ is perpendicular to $V Z_{1}$, then $k_{1}(\xi)=0$ a.e. and $k_{2}(\xi)=0$ a.e. This means that the linear hull of $V Z_{1}$ is dense in $L^{2}(-\infty, \infty) \oplus L^{2}(-\infty, \infty)$ and establishes our claim that $V$ is onto.

It is not difficult to check now that $V$ carries the spectral function of $A^{+}$into the spectral function of the multiplication operator in $L^{2}(-\infty, \infty) \oplus L^{2}(-\infty, \infty)$. Hence, if $\rho^{\prime}(\xi)>0$ for all $\xi, A^{+}$is unitarily equivalent to the multiplication operator in $L^{2}(-\infty, \infty)$ $\oplus L^{2}(-\infty, \infty)$.

Now suppose that $\rho^{\prime}(\xi)=0$ for all $\xi$. Then by equation (14), $\delta_{11}(\xi) \delta_{22}(\xi)-\delta_{12}^{2}(\xi)=0$ for all $\xi$. Equation (13) becomes

$$
\begin{aligned}
\left(\left[E^{+}(\beta)-\right.\right. & \left.\left.E^{+}(\alpha)\right] f, h\right) \\
= & \int_{\alpha}^{\beta}\left[C_{1}(f ; \xi) \delta_{11}^{1 / 2}(\xi)+\delta_{12}(\xi) \delta_{11}^{-1 / 2}(\xi) C_{2}(f ; \xi)\right] \\
& \cdot\left[C_{1}(h ; \xi) \delta_{11}^{1 / 2}(\xi)+\delta_{12}(\xi) \delta_{11}^{-1 / 2}(\xi) C_{2}(h ; \xi)\right]^{-} d \xi .
\end{aligned}
$$

In this case we define an operator $V$ on $Z_{1}$ into $L^{2}(-\infty, \infty)$ as follows:

$$
V\left[E^{+}(\beta)-E^{+}(\alpha)\right] f=\chi_{[\alpha, \beta)}(\xi)\left[C_{1}(f ; \xi) \delta_{11}^{1 / 2}(\xi)+\delta_{12}(\xi) \delta_{11}^{-1 / 2}(\xi) C_{2}(f ; \xi)\right] .
$$

Proceeding as above, we can use $V$ to show that $A^{+}$is unitarily equivalent to the multiplication operator in $L^{2}(-\infty, \infty)$. If $A^{+}$is a selfadjoint extension of $A$ in $H$, then $\rho(\xi) \equiv 0$, and the above holds.

This completes the proof of Theorem 2 .

\section{REFERENCES}

1. N. I. Achieser and I. M. Glasmann, Theorie der linearen Operatoren im HilbertRaum, Akademie-Verlag, Berlin, 1954.

2. R. C. Gilbert, Symmetric operators with singular spectral functions, Proc. Amer. Math. Soc. 21 (1969), 43-49.

3. M. A. Naimark, Spectral functions of a symmetric operator, Izv. Akad. Nauk SSSR Ser. Mat. 4 (1940), 277-318.

California State College at Fullerton 